\title{
Video Article \\ The Optokinetic Response as a Quantitative Measure of Visual Acuity in Zebrafish
}

\author{
Donald Joshua Cameron ${ }^{1,2}$, Faydim Rassamdana ${ }^{1}$, Peony Tam $^{1}$, Kathleen Dang ${ }^{1}$, Carolina Yanez ${ }^{3}$, Saman Ghaemmaghami ${ }^{3}$, Mahsa \\ Iranpour Dehkordi ${ }^{3}$ \\ ${ }^{1}$ College of Optometry, Western University of Health Sciences \\ ${ }^{2}$ Molecular Neurobiology Group, Western University of Health Sciences \\ ${ }^{3}$ Graduate College of Biomedical Sciences, Western University of Health Sciences
}

Correspondence to: Donald Joshua Cameron at jcameron@westernu.edu

URL: https://www.jove.com/video/50832

DOI: doi:10.3791/50832

Keywords: Neuroscience, Issue 80, Zebrafish, Eye Movements, Visual Acuity, optokinetic, behavior, adult

Date Published: 10/9/2013

Citation: Cameron, D.J., Rassamdana, F., Tam, P., Dang, K., Yanez, C., Ghaemmaghami, S., Dehkordi, M.I. The Optokinetic Response as a Quantitative Measure of Visual Acuity in Zebrafish. J. Vis. Exp. (80), e50832, doi:10.3791/50832 (2013).

\section{Abstract}

Zebrafish are a proven model for vision research, however many of the earlier methods generally focused on larval fish or demonstrated a simple response. More recently adult visual behavior in zebrafish has become of interest, but methods to measure specific responses are new coming. To address this gap, we set out to develop a methodology to repeatedly and accurately utilize the optokinetic response (OKR) to measure visual acuity in adult zebrafish. Here we show that the adult zebrafish's visual acuity can be measured, including both binocular and monocular acuities Because the fish is not harmed during the procedure, the visual acuity can be measured and compared over short or long periods of time. The visual acuity measurements described here can also be done quickly allowing for high throughput and for additional visual procedures if desired. This type of analysis is conducive to drug intervention studies or investigations of disease progression.

\section{Video Link}

The video component of this article can be found at https://www.jove.com/video/50832/

\section{Introduction}

Zebrafish (Danio rerio) are a good model to study visual physiology due to the similarity of their retina to other vertebrates, their short life cycle, and the availability of genetically altered mutants ${ }^{1,2}$. The optokinetic reflex/response/nystagmus (OKR) is a combination of smooth pursuit and rapid saccade eye movements. For more than 60 years clinicians have demonstrated that the OKR can be used for objectively measuring visual acuity in patients and is especially useful for determining infant visual capabilities ${ }^{3-5}$. The first recorded use of OKR in animals was using pigeons in the $1950 \mathrm{~s}^{6}$. More recently, the OKR has become a valuable tool for assessing the visual function in larval zebrafish and is often used to screen for genetic mutants that have a visual impairment ${ }^{1,2}$. While the OKR has been widely used to determine visual function in larval zebrafish, it has only recently been demonstrated in adult zebrafish ${ }^{7-10}$. A recent paper by Tappeiner et al. uses a commercially available optomotor system traditionally used for mice, OptoMotry, to demonstrate that the visual acuity of the adult zebrafish remains quite constant, $\sim 0.59$ cycles per degree (cpd), even across various angular velocities ${ }^{7}$

Although the OKR has proven to be quite useful in larval zebrafish studies, other methods for visual behavior have been used in zebrafish adults with varying degrees of success. The escape response assay demonstrated that albino and ruby zebrafish, both pigmentation mutants, have reduced visual responses in bright light conditions ${ }^{11,12}$. This same escape response assay has also successfully identified night blindness $d$ mutants, albeit at 2 years of age ${ }^{13}$. However, the escape response assay is not without flaws. It is difficult to ascribe to an exact visual function and is only a gross approximation of visual changes - meaning it takes a large change before the change is identified.

Another method that has been developed for identifying adult zebrafish with visual impairments is the optomotor response (OMR) ${ }^{11}$. In this assay, fish are placed in a circular tank with an opaque column in the center. Black and white stripes rotate around the tank and the fish is tasked with swimming in the direction of the stripe motion. Like the escape response, the OMR focuses on the visuomotor capabilities of the adult zebrafish. It has however been used successfully to identify fish with visual impairments, such as the Irp2/bugeye mutants ${ }^{14}$. The Irp2/bugeye zebrafish exhibit buphthalmos, elevated intraocular pressure, diminished OMR, and progressive retinal degeneration ${ }^{14,15}$.

Many studies have used the visuomotor capabilities in adult zebrafish, which are often subjective and cannot be quantitatively analyzed. By using the OKR, one can study the visual acuity of adult fish more objectively. We have built our own OKR device, modeled after one initially used for larval studies ${ }^{1}$. In this study we demonstrate that the OKR can be used to calculate both monocular and binocular visual acuity in zebrafish. 


\section{Protocol}

All animal husbandry and experiments should be approved and conducted in accordance with guidelines set forth by the Institutional Animal Care and Use Committee of the respective institution or other legal requirements.

\section{Zebrafish Care}

1. Maintain zebrafish under standard conditions at $28.5^{\circ} \mathrm{C}$ on a $10 \mathrm{hr}$ dark- $14 \mathrm{hr}$ light cycle ${ }^{16}$.

2. Keep adult zebrafish at a tank density of about $3 \mathrm{fish} / \mathrm{L}$.

\section{Optokinetic response (OKR)}

1. Build the custom OKR recording device using a $14.5 \mathrm{~cm}$ diameter rotating drum, a stereo microscope with adjustable light intensity settings (300-8,000 lux) and a computer (Figure 1a).

1. Attach a camera to the microscope that will provide a live feed onto an adjacent monitor and allow for image capture and recording.

2. Control the rotating drum using a microcontroller linked to a computer which will accommodate various speed and direction selections.

2. Anesthetize the fish in $0.016 \%$ tricaine for $2-3 \mathrm{~min}$ and then place the fish on a small platform with the eyes and gills suspended over the edge.

3. Place a thin sponge/towel over the body of the fish and pin 2-3 pieces of foam, shaped to accommodate the fish, over the fish to keep it immobilized. Limiting the movement of the tail of the fish was found to be a crucial aspect for immobilization without causing injury to the fish, and was best achieved using a flat piece of foam. (Figure 1b)

4. Position the fish within a cylindrical water-filled tank, that fits inside the rotating drum of the OKR recording device. Use magnets on the platform to position the fish upright, with the eyes approximately $7.3 \mathrm{~cm}$ from the edge of the drum. The fish should revive from the anesthesia within a few minutes - normal breathing will resume and random eye movements should be observed.

5. Place a base grating of 0.07 cycles per degree (cpd) into the rotating drum and engage the computer controls to begin rotation and video capture (a speed of $12 \mathrm{rpm}$ was used for this paper, but speeds of 8-16 rpm should give similar results).

6. Once an initial OKR is elicited by the base grating, pause the rotation briefly, and replace the grating with a smaller grating (higher spatial frequency).

7. Repeat this process until an OKR can no longer be elicited. Retest with the smallest grating that caused an OKR following a modified staircase approach and then repeat using the grating that failed to elicit a response to verify the loss of the OKR. This process may be repeated to verify a true response to the smallest detectable grating if the final grating changed relative to the first extinction event.

8. Obtain monocular acuity measurements by placing a black plastic occluder over the stripes adjacent to the opposite eye. Repeat the procedure above (steps 2.6-2.7).

9. Obtain the acuity of the opposite eye by repositioning the occluder and repeating the steps $2.6-2.7$ again.

10. Note the distance each eye is from the stripes during the testing procedure by using the reference measuring strip beneath the fish tank so that accurate acuity measures can be calculated in the following steps.

\section{Calculating Visual Acuity} Obtain the visual acuity by calculating the cpd: $\frac{1}{2 \tan ^{-1}\left(\frac{h}{2 a}\right)}$ where $a$ is the distance from the center of the lens to the grating, and $h$ is the length of one cycle of the smallest grating at which OKR was observed (Figure 1c).

2. For combined visual acuity measurements, the averaged a-value from both the left and right eye is used.

\section{Representative Results}

\section{OKR Device Functionality}

The OKR device as explained above and shown in Figure 1 functions with minimal maintenance. As noted in step 2.3, immobilizing the tail is critical to keeping the fish restrained during the recording. Sufficiently immobilized, the fish can be kept in the OKR device for prolonged periods of time. Consequently, care should be taken during the initial immobilization steps, so that more data can be collected for each individual fish.

\section{Visual acuity measurements}

The binocular OKR is readily elicited in normal adult fish. By following the procedure above one can find visual acuities for zebrafish of many ages (Figure 2). The best binocular spatial acuity we recorded was $0.74 \mathrm{cpd}$. As noted in Figure 2, the binocular acuity generally increases with age ( $n=46 ; p=0.002$ ANOVA using SPSS v14), but levels off after about 12 months of age. When we compared the measured visual acuity in 5 month old wild type fish to age-matched Irp2/bugeye fish, we found that the Irp2/bugeye fish had significantly reduced acuity ( $p<0.001 ;$ wild type: $0.49 \pm 0.05 \mathrm{cpd}$; $\mathrm{n}=8$; Irp2/bugeye: 0.35 $\pm 0.06 ; \mathrm{n}=10$; Student's T-test using Excel).

By occluding the right eye the visual acuity for the left eye can be determined and vice versa. When compared to the binocular acuity, binocular summation is observed similarly to human vision ${ }^{17}$ (Figure 3 ). The binocular acuity was generally $5-10 \%$ better than either right (OD) or left (OS) acuities measured independently. For some individual fish the difference was a $25 \%$ improvement. 


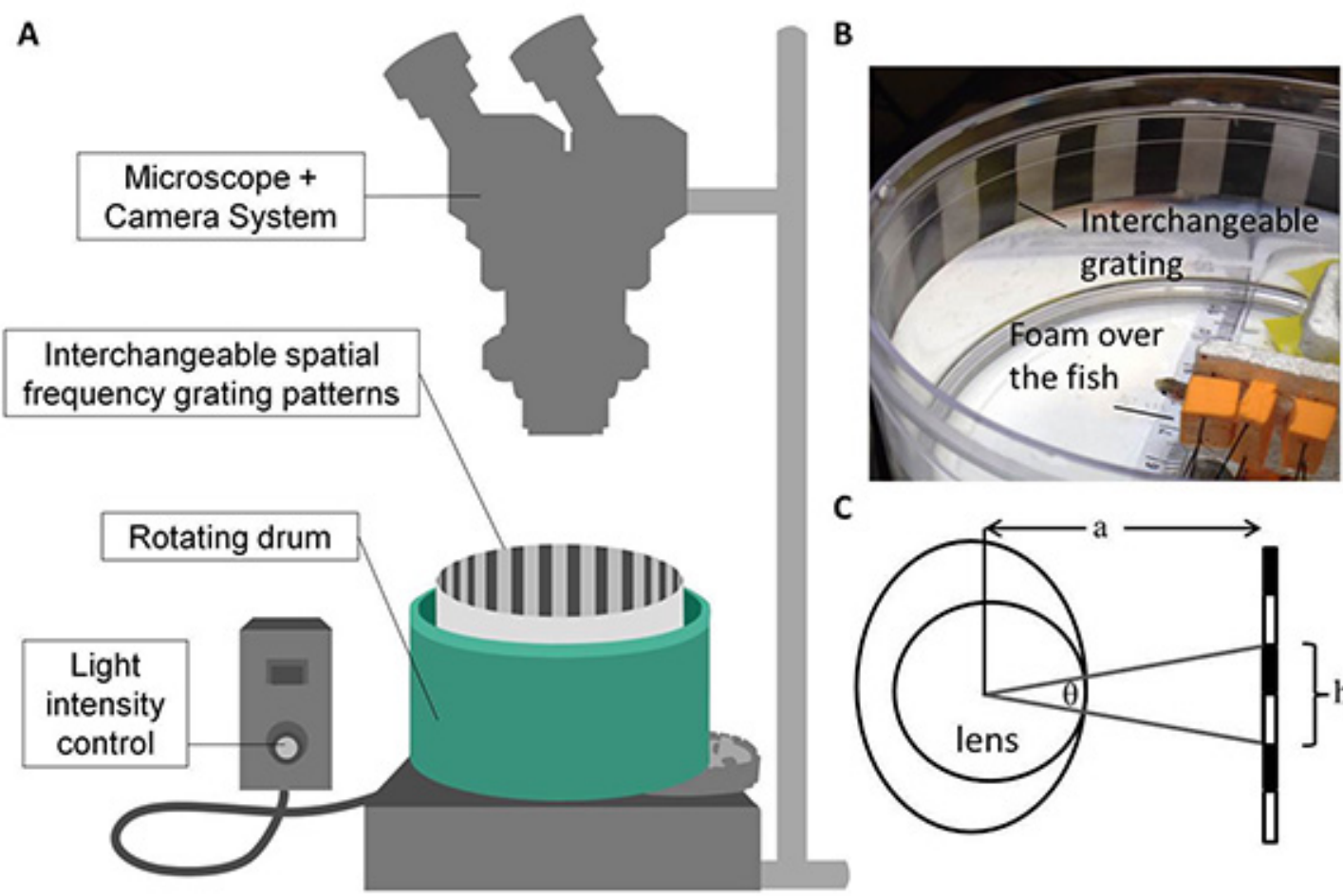

Figure 1. OKR recording. A) The OKR device consists of a $14.5 \mathrm{~cm}$ diameter rotating drum and microscope with adjustable light intensity settings (300-8,000 lux). A camera provides a live feed on an adjacent monitor. The rotating drum has various speed settings in both clockwise and counterclockwise directions and interchangeable spatial frequency gratings are inserted and removed as needed. B) Fish are secured and placed in the center of the rotating drum. C) Cycles per degree (cpd) is calculated using $\mathrm{cpd}=\frac{1}{2 \tan ^{-1}\left(\frac{h}{2 a}\right)}$ Click here to view larger image. 


\section{Visual Acuity Changes with Age}

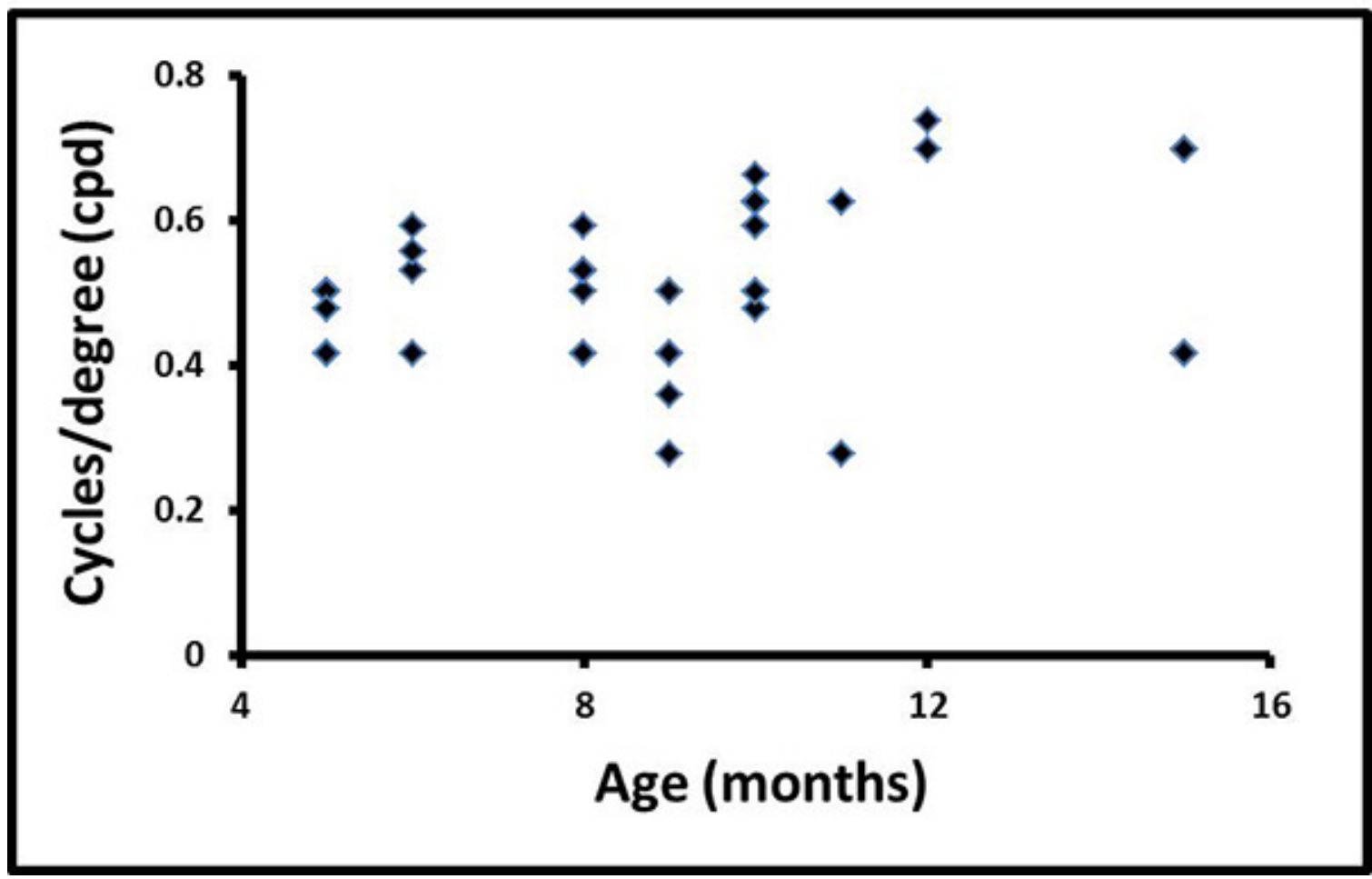

Figure 2. Visual acuity changes with age. The graph shows independent visual acuities measured 5-15 months in age. The visual acuity generally increases throughout the first year of development and then tails off a bit at 15 months of age ( $p=0.002$ ANOVA using SPSS v14). Each point may either represent 1 or several fish. Visual acuity is measured in cycles/degree (cpd). $(n=46$ total; 8 at 5 months; 4 at 6 months; 7 at 8 months; 6 at 9 months; 11 at 10 months; 2 at 11 months; 2 at 12 months; and 6 at 15 months). Click here to view larger image.

\section{Binocular Visual Acuity Summation Effect}

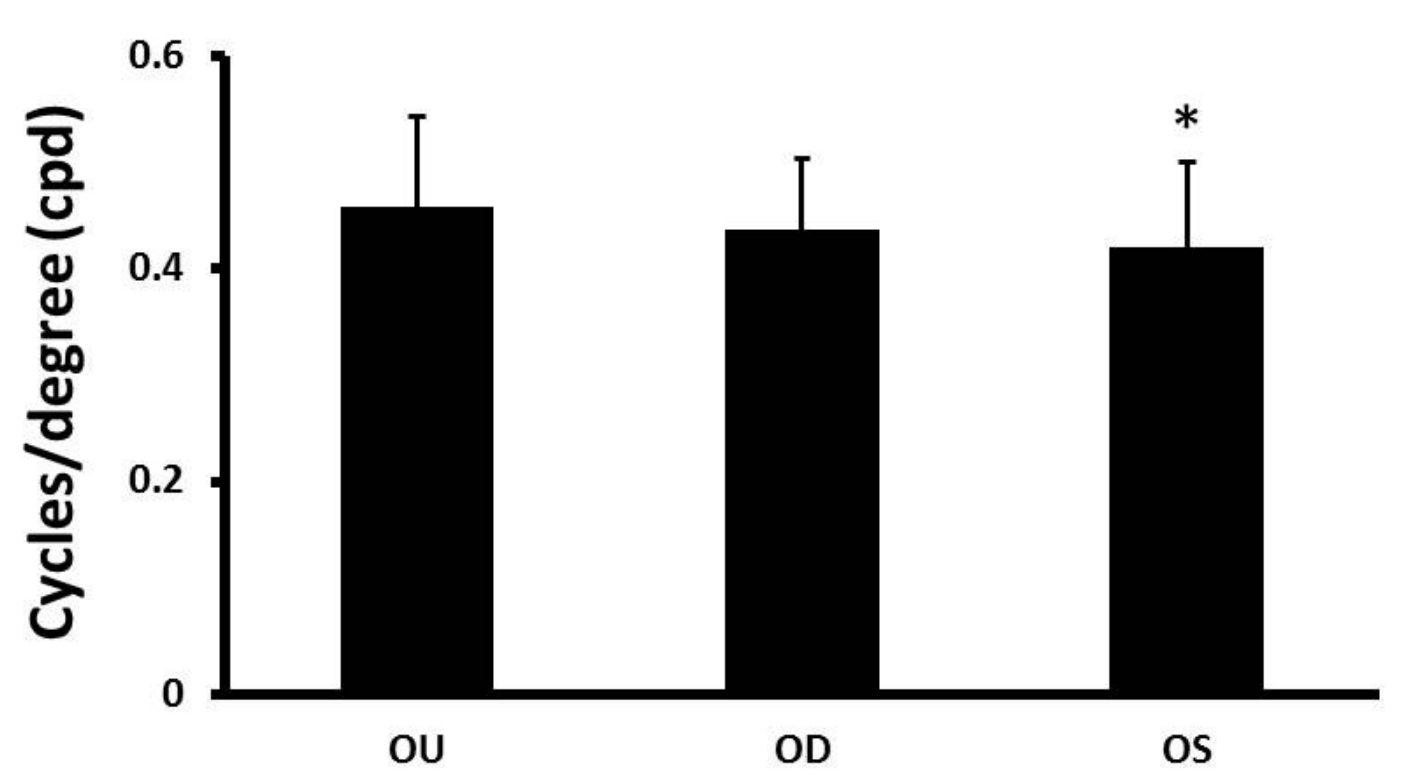

Figure 3. Binocular visual acuity summation effect. The binocular (OU) visual acuity is generally higher compared to right (OD) or left (OS) eye acuities. In this particular group the OS acuities were significantly lower. ( ${ }^{*} \mathrm{p}=0.006, \mathrm{~N}=8$ ) Click here to view larger image. 


\section{Discussion}

On occasion the fish may escape into the water tank. It is critical to immobilize the tail. Additionally the use of a thin sponge/towel over the fish's body was also found to be invaluable to keeping the fish suspended for prolonged periods of time. We were able to keep fish immobilized for more than 30 min using this procedure without harming the fish. This amount of time is more than sufficient to do the binocular and both monocular acuities.

The presentation of the grating can be either done as described here and elsewhere using a physically rotating drum or by presenting a digital display ${ }^{18-22}$. Both types have specific advantages and disadvantages. We chose to use the physical rotation both for cost reasons and technical specifications. Excluding the computer and imaging equipment, the OKR device described here can be built for around $\$ 150$.

The OKR is an objective means of determining visual acuity in adult zebrafish. While several other visuomotor assays for vision have been used in previous experiments and even the electroretinogram has been successfully done in adult zebrafish eyes, though it has not been heavily utilized, the adult zebrafish OKR opens the door to many new experimental conditions ${ }^{22}$. This study has demonstrated the OKR's utility in measuring visual acuity changes over time, identifying visual acuity difference in eye disease models, and in determining monocular visual acuity.

Interestingly, adult zebrafish vision when compared to human vision (20/20) is approximately 20/1,000 (when 0.60 cpd is converted to -0.3 logMAR). Although this may seem poor, the zebrafish eyes are very well suited for its environment. Despite the visual task of looking through 6.5 $\mathrm{cm}$ of water, a few millimeters of polycarbonate, and another centimeter of air, the fish perform quite well! We found that the adult zebrafish acuity is about $70 \%$ of what would be predicted given their photoreceptor spacing. ${ }^{23}$ Contrast that with optimal human performance $\sim 20 / 12$, which is about $80 \%$ of the predicted $20 / 10$, and OKR technique for measuring visual capability in adult zebrafish is impressive ${ }^{24,25}$.

The technique of measuring independent eye visual acuities would allow for studies in which one eye could be treated and the other used as a control. Additionally, fish with asymmetric eye development such as the Irp2/bugeye or fish that develop cataract could be monitored more accurately. It should be noted that care needs to be taken to accurately measure the distance from the eyes to the stripes and to occlude the full field of vision. This technique should open the door to more accurate and specific measures of zebrafish visual function.

\section{Disclosures}

The authors have nothing to disclose.

\section{Acknowledgements}

Funding provided by the Western University of Health Sciences College of Optometry to DJC. Special thanks to Irisa Tam for graphic design.

\section{References}

1. Brockerhoff, S. E. et al. A behavioral screen for isolating zebrafish mutants with visual system defects. Proc. Natl. Acad. Sci. U.S.A. 92, 10545-10549 (1995).

2. Neuhauss, S. C. et al. Genetic disorders of vision revealed by a behavioral screen of 400 essential loci in zebrafish. J. Neurosci. 19, 8603-8615 (1999).

3. Schumann, W. P. The objective determination of visual acuity on the basis of the optokinetic nystagmus. Am. J. Optom. Arch. Am. Acad. Optom. 29, 575-583 (1952).

4. Gorman, J. J., Cogan, D. G. \& Gellis, S. S. An apparatus for grading the visual acuity of infants on the basis of opticokinetic nystagmus. Pediatrics. 19, 1088-1092 (1957).

5. Fulton, A. B., Manning, K. A. \& Dobson, V. A behavioral method for efficient screening of visual acuity in young infants. II. Clinical application. Invest. Ophthalmol. Vis. Sci. 17, 1151-1157 (1978).

6. Huizinga, E. \& Van Der Meulen, P. Vestibular rotatory and optokinetic reactions in the pigeon. Ann. Otol. Rhinol. Laryngol. 60, 927-947 (1951).

7. Tappeiner, C. et al. Visual Acuity and Contrast Sensitivity of Adult Zebrafish. Front. Zool. 9, 10, doi:1742-9994-9-10 [pii] 10.1186/1742-9994-9-10 (2012).

8. Mueller, K. P., Schnaedelbach, O. D., Russig, H. D. \& Neuhauss, S. C. VisioTracker, an innovative automated approach to oculomotor analysis. J. Vis. Exp. (56), e3556, doi:10.3791/3556 3556 [pii] (2011).

9. Zou, S. Q. et al. Using the optokinetic response to study visual function of zebrafish. J. Vis. Exp. (36), e1742, doi:10.3791/1742 1742 [pii] (2010).

10. Mueller, K. P. \& Neuhauss, S. C. Quantitative measurements of the optokinetic response in adult fish. J. Neurosci. Methods. 186, 29-34, doi:S0165-0270(09)00592-5 [pii] 10.1016/j.jneumeth.2009.10.020 (2010).

11. Li, L. \& Dowling, J. E. A dominant form of inherited retinal degeneration caused by a non-photoreceptor cell-specific mutation. Proc. Natl. Acad. Sci. U.S.A. 94, 11645-11650 (1997).

12. Ren, J. Q., McCarthy, W. R., Zhang, H., Adolph, A. R. \& Li, L. Behavioral visual responses of wild-type and hypopigmented zebrafish. Vision Res. 42, 293-299, doi:S004269890100284X [pii] (2002).

13. Maaswinkel, H., Mason, B. \& Li, L. ENU-induced late-onset night blindness associated with rod photoreceptor cell degeneration in zebrafish. Mech. Ageing Dev. 124, 1065-1071, doi:S0047637403001921 [pii] (2003).

14. Stujenske, J. M., Dowling, J. E. \& Emran, F. The bugeye mutant zebrafish exhibits visual deficits that arise with the onset of an enlarged eye phenotype. Invest. Ophthalmol. Vis. Sci. 52, 4200-4207, doi:iovs.10-6434 [pii] 10.1167/iovs.10-6434 (2011). 
15. Veth, K. N. et al. Mutations in zebrafish Irp2 result in adult-onset ocular pathogenesis that models myopia and other risk factors for glaucoma. PLoS Genet. 7, e1001310, doi:10.1371/journal.pgen.1001310 (2011).

16. Westerfield, M. THE ZEBRAFISH BOOK, A guide for the laboratory use of zebrafish (Danio rerio). 5th edn, University of Oregon Press (2007).

17. Cagenello, R., Arditi, A. \& Halpern, D. L. Binocular enhancement of visual acuity. J. Opt. Soc. Am. A Opt. Image Sci. Vis. 10, 1841-1848 (1993).

18. Brockerhoff, S. E. Measuring the optokinetic response of zebrafish larvae. Nat. Protoc. 1, 2448-2451, doi:10.1038/nprot.2006.255 (2006).

19. Hodel, C. \& Neuhauss, S. C. Computer-based analysis of the optokinetic response in zebrafish larvae. CSH Protoc. 2008, pdb prot4961, doi:10.1101/pdb.prot4961 (2008).

20. Huber-Reggi, S. P., Mueller, K. P. \& Neuhauss, S. C. Analysis of optokinetic response in zebrafish by computer-based eye tracking. Methods Mol. Biol. 935, 139-160, doi:10.1007/978-1-62703-080-9_10 (2013).

21. Tappeiner, C. et al. Visual acuity and contrast sensitivity of adult zebrafish. Front. Zool. 9, 10, doi:10.1186/1742-9994-9-10 (2012).

22. Saszik, S. \& Bilotta, J. The effects of temperature on the dark-adapted spectral sensitivity function of the adult zebrafish. Vision Res. 39 , 1051-1058, doi:S0042-6989(98)00237-5 [pii] (1999).

23. Haug, M. F., Biehlmaier, O., Mueller, K. P. \& Neuhauss, S. C. Visual acuity in larval zebrafish: behavior and histology. Front. Zool. 7, 8 , doi:1742-9994-7-8 [pii] 10.1186/1742-9994-7-8 (2010).

24. Curcio, C. A., Sloan, K. R., Kalina, R. E. \& Hendrickson, A. E. Human photoreceptor topography. J. Comp. Neurol. 292, 497-523, doi:10.1002/ cne.902920402 (1990).

25. Kalloniatis, M. \& Luu, C. Visual Acuity (1995) 